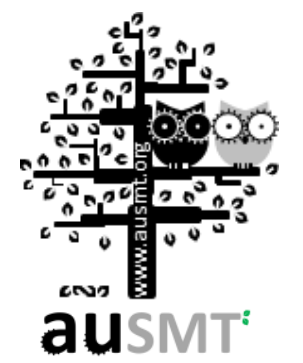

\title{
Analysis of Personal Life Patterns Using Accelerometer-Based Wearable Devices
}

\author{
Yeh-Liang Hsu', 2, *, Yi-Fan Jiang1, ${ }^{1}$, Ching-Hung Lin ${ }^{3}$, Tsai-Ya Lai ${ }^{4}$, and Cheng- \\ Li Chang ${ }^{4}$
}

${ }^{1}$ Department of Mechanical Engineering, Yuan Ze University, Taoyuan 32003, Taiwan

${ }^{2}$ Gerontechnology Research Center, Yuan Ze University, Taoyuan 32003, Taiwan

${ }^{3}$ Physical Education Office, Yuan Ze University, Taoyuan 32003, Taiwan

${ }^{4}$ Service Systems Technology Center, Industrial Technology Research Institute, Hsinchu 31040, Taiwan

(Received 23 May 2015; Accepted 22 July 2015; Published on line 1 December 2015)

*Corresponding author: mehsu@saturn.yzu.edu.tw

DOI: $10.5875 /$ ausmt.v5i4.960

\begin{abstract}
Many commercially available wearable devices are equipped with sensors to measure motion and physiological signals from the wearer. G-sensors are commonly used in such wearable devices for counting steps, estimating energy expenditure and detecting sleep duration. In this study, two features derived from G-sensor motion signals, average cadence (step count divided by time) and ratio of high $\mathrm{G}$ value (outside the range of $0.5 \mathrm{~g} \sim 1.5 \mathrm{~g}$ ), were used to classify physical activities into four intensity levels (sedentary, light, moderate, hard). Eighty physical activity samples were collected and trained by the Weka machine learning software to form a classification model. G-sensor motion signals from four participants were collected over two weeks and classified into four activity intensity levels using the model. Physical activity levels (PAL) and personal life patterns of the participants were then derived. This data can then be used to tailor additional services for individual users of wearable devices. A BLE (Bluetooth Low Energy) based system for older adults with dementia, combining personal life pattern analysis with localization function, is also proposed as an example application.
\end{abstract}

Keywords: Wearable device, motion signal, activity intensity level, physical activity level

\section{Introduction}

Wearable devices are becoming increasingly popular. Many commercially available wearable devices are equipped with sensors to measure motion and physiological signals of the users. G-sensors are commonly used in such wearable devices to measure motion signals from the user. Such devices are less restricted in measuring positions and do not require electrodes to touch the skin, providing increased convenience and design flexibility and making them popular for use in wearable devices for counting steps and detecting sleep duration.

Such wearable devices often work with mobile device applications (Apps) for further data processing and display. Algorithms based on step count, and the user's height and weight are used to estimate travel distance and energy expenditure. However, estimations based on step count are relatively inaccurate. For example, energy expenditure is assumed to be zero for activities with zero step count, while walking and running are treated as having identical energy expenditure for the same number of steps, though the intensities of the two activities are very different. In other words, physical activity intensity (and therefore energy expenditure) cannot be correctly classified based on step count alone.

Physical activity intensity can be classified into four levels: sedentary, light, moderate, and hard [1], and can be accurately measured by calculating the calories burnt from the amount of oxygen uptake. However, this method can be only be implemented through the use of a cardiopulmonary motion detection system in a laboratory setting. On the other hand, the "heart rate reserve" 
method is often used to estimate the level of physical activity intensity based on the user's heart rate while performing a given physical activity. The heart rate reserve percentage is calculated by Eq. (1). This percentage can be used to determine physical activity intensities from Table 1 [2], Heart rate reserve $(\%)=$

$$
\left(H R-H R_{\text {rest }}\right) /\left(H R_{\text {max }}-H R_{\text {rest }}\right)
$$

where HR is the user's heart rate measured while performing a given physical activity, $\mathrm{HR}_{\max }=220$-Age, and $H R_{\text {rest }}$ is the heart rate measured after the user rests for five minutes. Note that age and individual differences are taken into account in the heart rate reserve method through $\mathrm{HR}_{\max }$ and $\mathrm{HR}_{\text {rest. }}$.

\begin{tabular}{|c|c|}
\hline Intensity & Heart rate reserve (\%) \\
\hline Very light & $<20$ \\
\hline Light & $20-39$ \\
\hline Moderate & $40-59$ \\
\hline Hard & $60-84$ \\
\hline Very hard & $>85$ \\
\hline
\end{tabular}

Table 1. Heart rate reserve to estimate activity intensity [2].

The purpose of this study is to interpret users' motion signals measured from G-sensors as physical activity intensity. Two features derived from the G-sensor motion signals, average cadence (step count divided by time) and ratio of high $G$ value (outside the range of $0.5 \mathrm{~g} 1.5 \mathrm{~g}$ ), were used to classify the physical activities into four intensity levels. Eighty physical activity samples were collected and trained by Weka machine learning software [3] to construct a classification model which can predict physical activity intensity from these two features. From the physical activity intensity, total daily energy expenditure (TDEE), physical activity levels (PAL) and personal life patterns can be derived. Further services can then be tailored for the wearable device user based on the data provided by wearable devices.

Senior citizens suffering from dementia were asked to wear GPS based wearable devices or RFID tags, mainly for positioning or localization purposes. At the end of the paper, a Bluetooth Low Energy (BLE) based system for older adults, combining personal life pattern analysis with basic localization function, is proposed as an application

YEH-LIANG HSU is a professor in the Department of Mechanical Engineering Yuan Ze University. He is also director of Gerontechnology Research Center.

YI-FAN JIANG is a graduate student in the Department of Mechanical Engineering, Yuan Ze University.

CHING-HUNG LIN is an associate professor and director of the Physical Education Office, Yuan Ze University.

TSAI-YA LAI is Business Director of Western Europe Office, Industrial Technology Research Institute, Taiwan.

CHENG-LI CHANG is an engineer in the Healthcare Services Division, Service Systems Technology Center, Industrial Technology Research Institute, Taiwan.

Copyright (C) 2015 International Journal of Automation and Smart Technology example.

\section{Methodology}

Activity samples collection

As mentioned earlier, after some preliminary trials, two features derived from the motion signals sensed by G-sensors were selected to classify activity intensity: average cadence (step count divided by time) and ratio of high $\mathrm{G}$ value (outside the range of $0.5 \mathrm{~g} \sim 1.5 \mathrm{~g}$ ). In general, cadence and ratio of high $G$ value measured from sedentary activities are lower than those measured from light/moderate activities. The ratio of high $\mathrm{G}$ value helps to distinguish a moderate activity (e.g. fast walking) from a hard activity (e.g. running or jumping rope) which have similar cadences, while certain hard activities (e.g. bicycling) have a low ratio of high $G$ value but a high cadence.

As shown at the top of Fig. 1, activity samples were collected from 10 participants (six males and four females, aged 22 to 27 years old, average 24.2 years old). Each participant was asked to perform eight different physical activities at four different levels of intensity, for a total of 80 samples. Sedentary level activities included using a computer (c) and watching TV (t); light level activities included walking (w) and housework (h); moderate level activities included bicycling (b) and fast walking (fw); and hard level activities included running ( $r$ ) and jumping rope (j). When performing a physical activity, the heart rate of the participant was monitored. Two-minute activity samples were recorded only after confirming that the heart rate of the participant has reached the required intensity level as defined by the heart rate reserve method described earlier. The participant also carried a mobile phone with a special App installed to record step count and $G$ value (sampling rate $5 \mathrm{~Hz}$ ). These 80 activity samples (two minutes each) are plotted in Fig. 1 (top) using the average cadence and percentage of high $G$ value as the $x$ and $y$ axes. This figure shows clear distinctions between the data points for sedentary level (yellow), light level (green) and moderate level (blue) The data points for moderate level and hard level (red) are less distinguishable.

\section{Machine learning with Weka}

These activity samples were then input into the Weka machine learning software [3]. In addition to the 80 activity samples, 20 "rest" samples were also added. For the "rest" samples, the percentage of the higher $\mathrm{G}$ value and average cadence were both considered as zero. Naive Bayes Classifier was selected for machine learning, and 10 -fold cross validation was used to assess the machine 
learning result. The machine learning model's predictive accuracy was $94 \%$. Certain errors occurred in the region between moderate and hard activities.

Figure 1 (bottom) shows a visual display of the machine learning model which can predict the activity intensity from an activity data sample's cadence and percentage of high $\mathrm{G}$ value. Note that the data point in purple at the origin represents "rest". Only two features were used in this model, making it easily implemented in wearable devices. Figure 1 (bottom) can be further updated if more activity samples are collected.

Figure 2 shows a real life example from a 27 year-old male office worker chosen from the 10 participants. The mobile App was used to collect motion signals over a 24hour period including the participant's typical working day.
The average cadence and percentage of the high $\mathrm{G}$ value were recorded every 5 minutes. The data samples were classified by the model in Fig. 1. As shown in Fig. 2, in the 24-hour period, "rest" activity accounted for a total of 510 $\min$ (35.54\% of total activity), as opposed to $865 \mathrm{~min}$ (59.93\%) for "sedentary" activity, $45 \mathrm{~min}$ (3.14\%) for "light" activity, 10 min (0.70\%) each for "moderate" and "hard" activity.

Matching measurements with the participant's real activity log show he woke up around 8:30 a.m., went to office and stayed sedentary for the whole day. He went at jogging around 4:30 pm in the afternoon, had dinner with some friends and went to bed around 12 midnight.
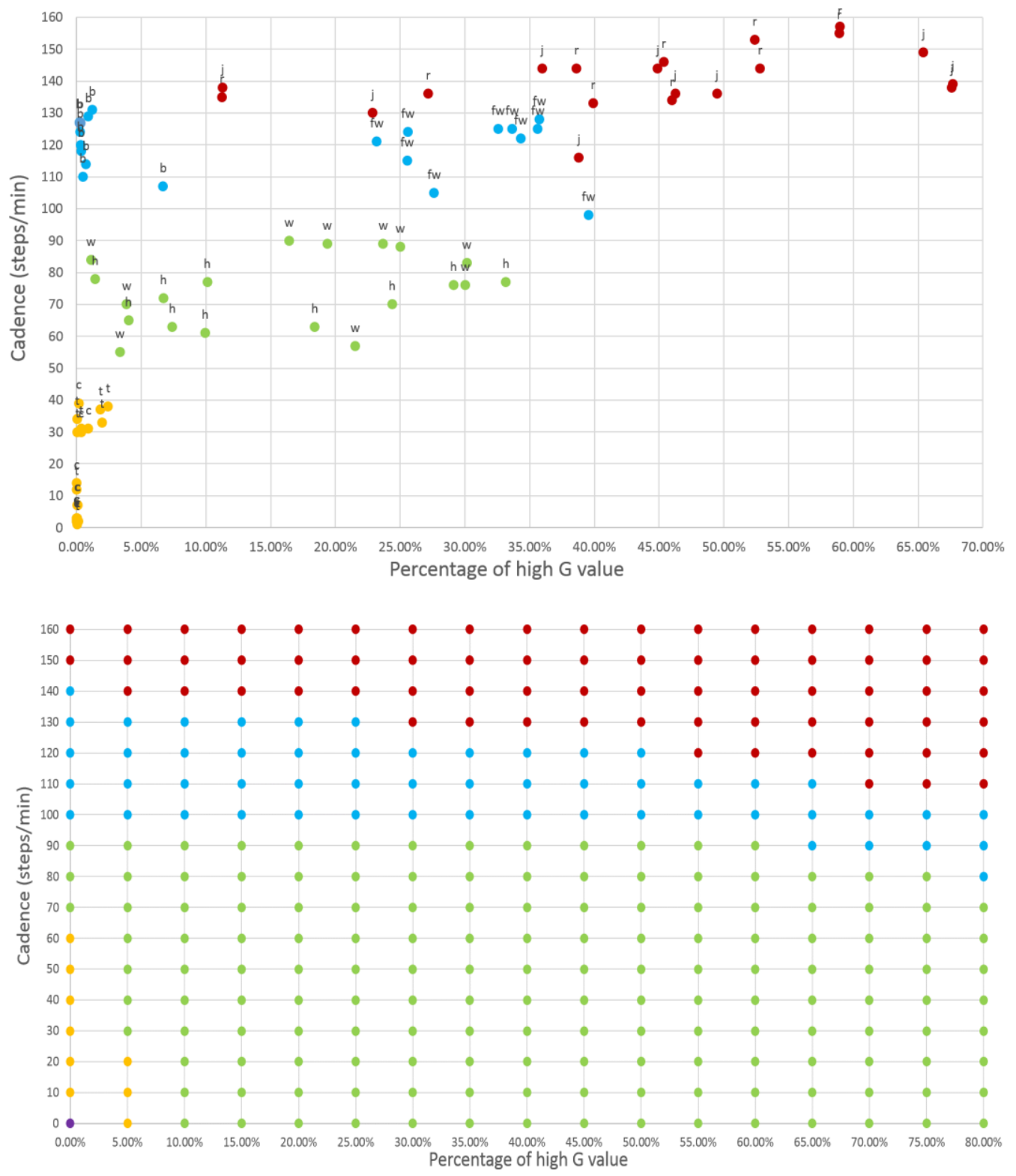

Figure 1. Eighty activity samples and the resulting visual display of machine learning models. 


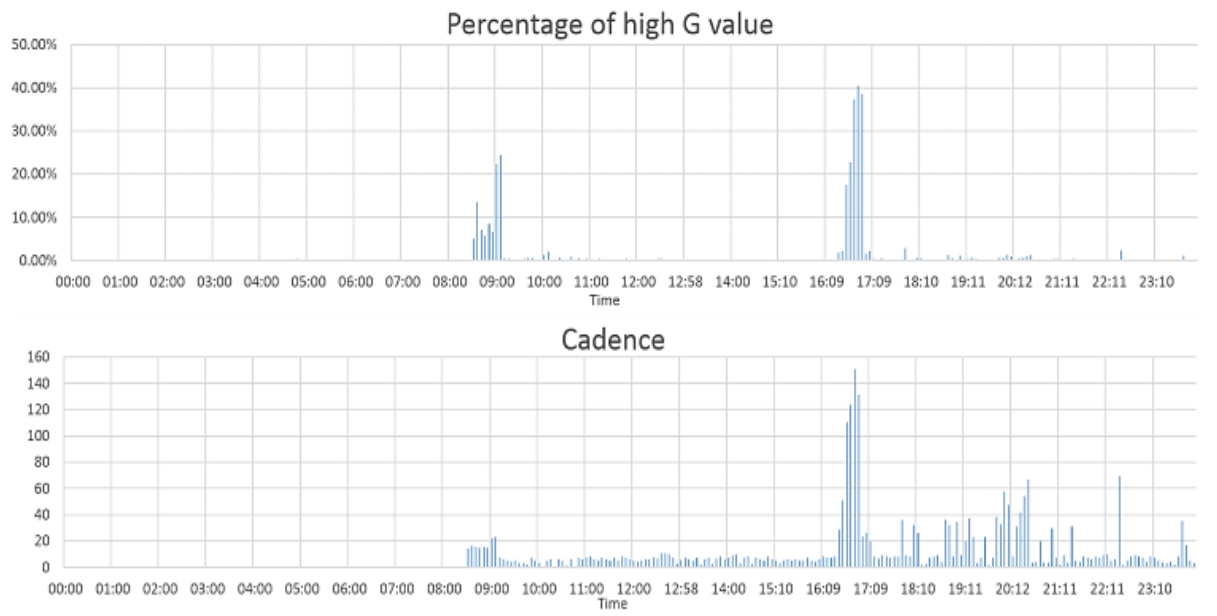

Activity Intensity

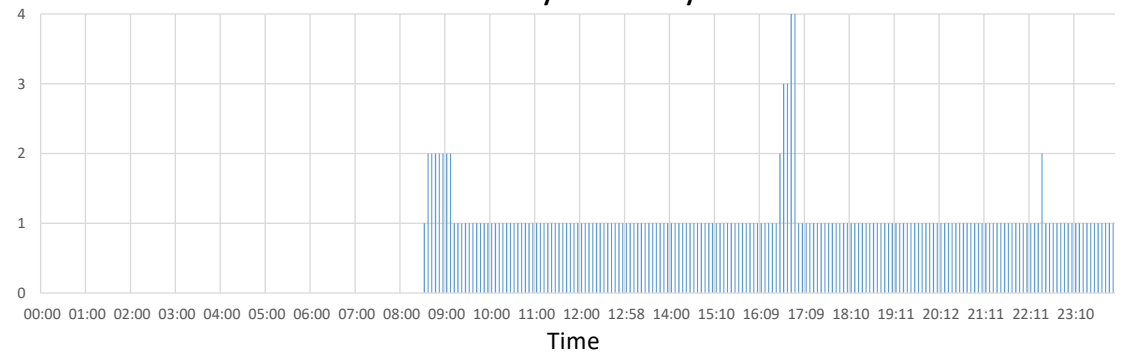

Figure 2. High G values and cadence over a whole day.

\section{Estimation of PAL (Physical Activity Level)}

PAL is often used as a daily lifestyle index:

$$
P A L=T D E E / B M R
$$

where TDEE is Total Daily Energy Expenditure (in calories), and BMR means Basal Metabolic Rate, defined as the minimal rate of energy expenditure per unit time when a person is at rest. BMR is estimated by Harris-Benedict equations, which was proposed in 1919 and amended in 1984 [4]:

Male:

BMR $=(13.397 \times w)+(4.799 \times h)-(5.677 \times a)+88.362$

Female:

$\mathrm{BMR}=(9.247 \times w)+(6.25 \times h)-(4.330 \times a)+447.593$

where $w$ is weight (in $\mathrm{kg}$ ), $h$ is height (in $\mathrm{cm}$ ), and $a$ is age. For example, the participant in Fig. 2 is a male, $173 \mathrm{~cm}, 60$ $\mathrm{kg}$, and 27 years old. His BMR is calculated as follows:

$(13.397 \times 60)+(4.799 \times 173)-(5.677 \times 27)+88.362$ $=1569.13(\mathrm{kcal})$

MET (Metabolic Equivalent of Task) is a physiological measure of energy expenditure of physical activities of different intensities. Table 2 compares the MET of different physical activities and ages. The unit of MET is $\mathrm{kcal} / \mathrm{kg} \cdot \mathrm{hr}$.

\begin{tabular}{|c|c|c|c|c|}
\hline \multicolumn{5}{|c|}{ Absolute intensity (METs) in healthy adults (age in years) } \\
\hline Type & $\begin{array}{c}\text { Young } \\
(28 \sim 39 y r)\end{array}$ & $\begin{array}{c}\text { Middle- } \\
\text { aged } \\
\left(40^{\sim} 64 \mathrm{yr}\right)\end{array}$ & $\begin{array}{c}\text { Old } \\
(65 \sim 79 y r)\end{array}$ & $\begin{array}{c}\text { Very-old } \\
(80 \sim \mathrm{yr})\end{array}$ \\
\hline Rest & 1 & 1 & 1 & 0.2 \\
\hline Sedentary & 1.8 & 1.8 & 1.4 & 0.8 \\
\hline Light & 2.4 & 2 & 1.6 & 1.1 \\
\hline Moderate & 4.8 & 4 & 3.2 & 2 \\
\hline Hard & 7.2 & 6 & 4.8 & 3 \\
\hline
\end{tabular}

According to Table 2, the TDEE of the 27-year old participant in Fig. 2 can be estimated as follows:

TDEE $=1.0 \times 60 \times 35.54 \% \times 24+1.8 \times 60 \times 59.93 \% \times 24$

$$
+2.4 \times 60 \times 3.14 \% \times 24+4.8 \times 60 \times 0.70 \% \times 24
$$$$
+7.2 \times 60 \times 0.70 \% \times 24=2294.64 \mathrm{kcal}
$$

Finally, the PAL of the participant for that particular day can be calculated:

$\mathrm{PAL}=$ TDEE $/ \mathrm{BMR}=2294.64 / 1569.13=1.46$

From the PAL, we can conclude that the participant had a sedentary lifestyle (see Table 3).

Table 2. MET of different physical activities and ages [5].

\begin{tabular}{|c|l|c|}
\hline Type & \multicolumn{1}{|c|}{ Lifestyle and level of activity } & PAL \\
\hline Inactive & Chair-bound or bed-bound & $1.2-1.4$ \\
\hline Sedentary & $\begin{array}{l}\text { Seated work with no option of moving around } \\
\text { and little or no strenuous leisure activity }\end{array}$ & $1.4-1.6$ \\
\hline Light & $\begin{array}{l}\text { Seated work with discretion and requirement } \\
\text { to move around but little or no strenuous } \\
\text { leisure activity }\end{array}$ & $1.6-1.8$ \\
\hline Moderate & Standing work (e.g. housework, shop assistant) & $1.8-2.0$ \\
\hline Hard & Strenuous work or highly active leisure & $2.0-2.4$ \\
\hline
\end{tabular}

Table 3. Lifestyle and level of activity [5]. 


\section{Results}

\section{Long-term analysis of four participants}

Four participants were recruited for long-term analysis of personal lifestyles: a college student (20 years old), two office workers (22 and 31 years old), and a clothing store owner (58 years). All four participants are males.

The four participants were asked to carry a mobile phone with our G-sensor App installed for 14 days. Average cadence and percentage of high $G$ value were extracted from the motion signals every 5 minutes. The data were then classified into four physical activity intensities. TDEE and PAL were calculated, and finally the lifestyle of that particular day was determined. The participants were also asked to keep a daily activity log.

Figures 3-6 display some representative daily data. Lifestyle differences among the four participants can be determined from the figures.

Participant A: office worker, 22 years old, $180 \mathrm{~cm}, 78 \mathrm{~kg}$, $B M R=1,872$

Participant A was an ordinary office worker with regular working hours. On the particular day shown in Fig. 3 , he was involved in sedentary activity level during most of his work time. He went to the gym in the evening and exercised for two hours. Thus that particular day was classified as sedentary. The daily PAL was around 1.6 to 1.9, denoting a moderately active lifestyle.

Participant B: college student, 20 years old, $174 \mathrm{~cm}, 65 \mathrm{~kg}$, $B M R=1,681$

Participant B was a college student. As shown in Fig. 4 , he woke up later than the office worker. He was engaged in moderate intensity level activity during lunch and dinner, but sedentary / light intensity level during most of rest of the day. The PAL on that particular day was 1.46 , and the lifestyle was "sedentary".

Participant C: clothing store owner, 58 years old, $175 \mathrm{~cm}$, $66 \mathrm{~kg}, B M R=1,483$

Participant $C$ was 58 years old and his $B M R$ value was lower than the other younger participants. On the day displayed in Fig. 4, he woke up at around 6:00 am, went for jogging for one hour at 7:00 am, and reached hard intensity for 38 minutes. He is a clothing store owner and was required to stand up and walk around the store during most of his working time, frequently achieving light to moderate activity intensity. He took an afternoon nap for about 1.5 hours, and went to bed at around 23:00. His PAL on the day shown in Fig. 5 was 1.84, and his lifestyle was "moderate".
Participant D: IC engineer, 31 years old, $172 \mathrm{~cm}, 64 \mathrm{~kg}$, $B M R=1595$

Participant D was a semiconductor plant engineer. On the day displayed in Fig. 6, he went to bed at 1:00 am and woke up at 8:00 am, engaged in light to moderate intensity activity while traveling to work (around 9:00), lunch time (12:00 13:00) and returning home (around 20:00). He took an afternoon nap for about 0.5 hours. He is required to stay standing during work time, frequently achieving light intensity activity during working hours. He went jogging for one hour around 21:00. His PAL on the day shown in Fig. 6 was 1.79, and his lifestyle was "light".

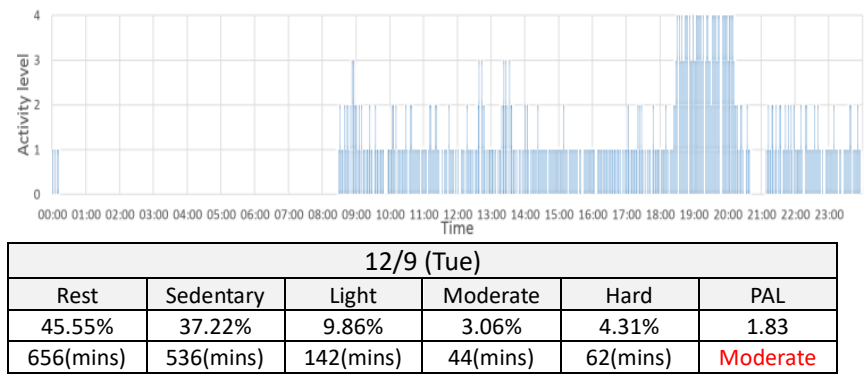

Figure 3. Activity intensity graph of participant A on 12/9.

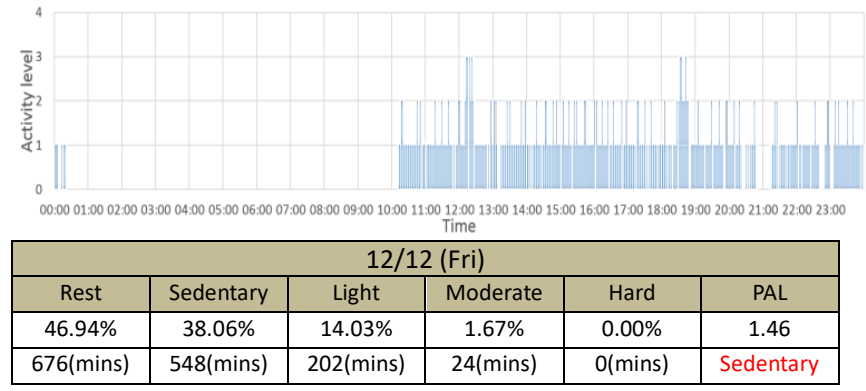

Figure 4. Activity intensity graph of participant B on 12/12.

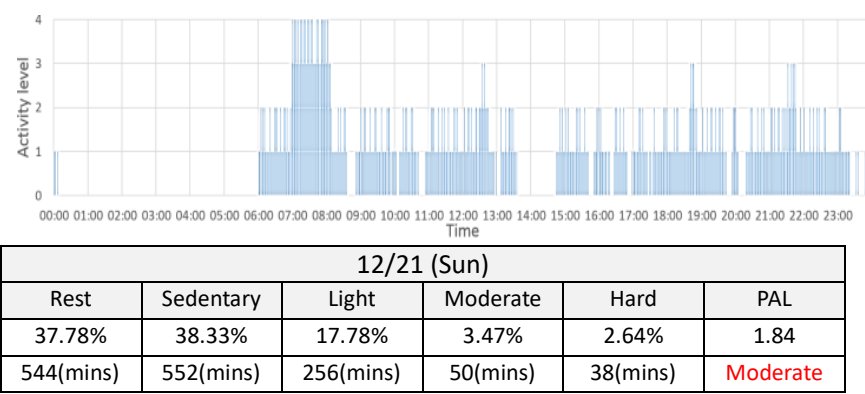

Figure 5 . Activity intensity graph of participant C on 12/21.

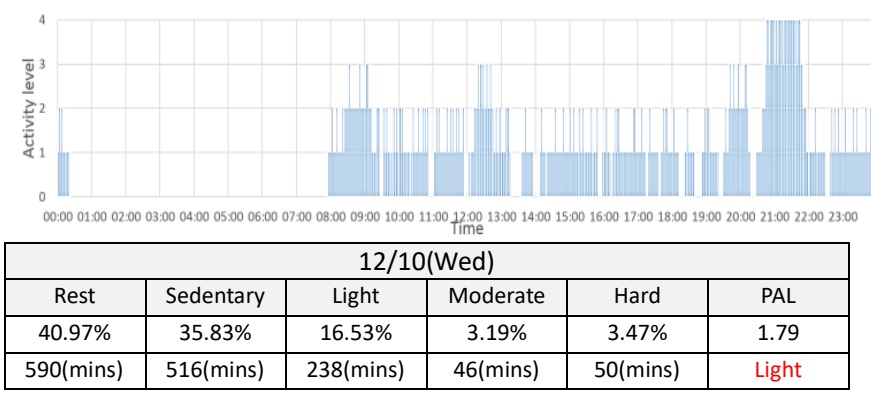

Figure 6. Activity intensity graph of participant D on 12/10. 


\section{Conclusion and future work}

According to the International Data Corporation (IDC) Worldwide Quarterly Wearable Device Tracker published in June 2015 (http://www.idc.com/tracker/), vendors shipped a total of 11.4 million wearable devices in 1Q15, a 200.0\% increase from the 3.8 million wearables shipped in 1Q14. Fitbit (https://www.fitbit.com/) accounted for $34.2 \%$ of total sales, followed by Miband (http://www.mi.com/sg/miband/) at 24.6\%. Both Fitbit and Mi-Band provide functions for counting steps, estimating energy expenditure and detecting sleep duration, based on G-sensor.

This paper proposes how physical activity intensity levels and lifestyle patterns can be derived from G-sensor motion signals. Further services can then be tailored for the user to generate value for the data provided by wearable devices. Currently, the types of activities used in the collected dataset are still limited. More features from G-sensor motion signals should be explored to account for a full range of daily activities.

The experiments performed in this study used a mobile App to collect motion signals from the G-sensor. Many individuals suffering from dementia wear GPSbased wearable devices or RFID tags to prevent them from getting lost. In addition to positioning or localization purposes, physical activity intensity levels and lifestyle patterns of the older adults can be monitored if existing G-sensor based wearable devices are used (e.g., Mi-Band).

The Mi-band uses Bluetooth low energy (BLE), a new class of wireless personal area network technology. Compared to classic Bluetooth, BLE offers considerably reduced power consumption and cost while maintaining a similar communication range. Mi-Band uses BLE proximity sensing to transmit a universally unique identifier which can be picked up by a compatible reader (app or operating system). The identifier can then be looked up to determine the device's physical location.

Figure 7 proposes a BLE-based system combining personal life pattern analysis with a localization function. This system consists of three parts: tag, reader, and server. The tag is a BLE wearable device with a built-in $\mathrm{G}$ sensor (namely, Mi-Band). Readers (such as Arduino Yun combined with $\mathrm{Hm}-10$ BLE module as shown in Fig. 7) are installed in the home or nursing facility. As a user wearing the tag approaches a reader, the tag's UUID, MAC address and RSSI are uploaded to the cloud server, allowing for the location of the user to be determined. In applications such as access control, the cloud server can also connect to the Google Cloud Messaging (MCG) service to immediately send an alert to the caregiver's Android device. In the meantime, motion signals from the $\mathrm{G}$ sensor are uploaded and converted to physical activity intensity levels and lifestyle patterns as previously described, which greatly adds value to such a system.

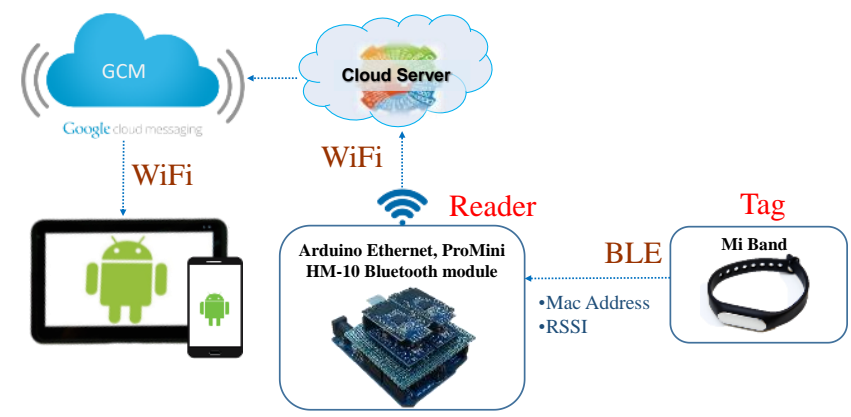

Figure 7. BLE-based system structure.

\section{References}

[1] The Health Promotion Administration, Ministry of Health and Welfare. "Activity intensity."

Available at:

http://www.hpa.gov.tw/Bhpnet/Web/Act/Strength OfSport.aspx, 2014

[2] M. Pollock, G. Gaesser, J. Butcher, J. Despres, R. Dishman, B. Franklin, and C. Garber, "ACSM Position Stand: The Recommended Quantity And Quality Of Exercise For Developing And Maintaining Cardiorespiratory And Muscular Fitness, And Flexibility In Healthy Adults," Medicine \& Science in Sports \& Exercise, vol. 30, no. 6, pp. 975-991, 1998. doi: 10.1097/00005768-199806000-00032

[3] Machine Learning Group at the University of Waikato. "Weka 3: Data Mining Software in Java." Available at: http://www.cs.waikato.ac.nz/ml/weka/, 2014

[4] A. Roza and H. Shizgal, "The Harris Benedict equation reevaluated," American Journal of Clinical Nutrition, vol. 40, no. 1, pp. 168-182, 1984. doi: $10.1007 / B F 02998927$

[5] N. Scrimshaw, "Energy and protein requirements," in proceedings of an IDECG workshop held at the Lochipdalendon School of Hygiene and Tropical Medicine, Stockton, UK, 1994. doi: $10.1097 / 00001888-193111000-00026$ 